\title{
Linx
}

Revue des linguistes de l'université Paris X Nanterre

$50 \mid 2004$

Variation sémantique et syntaxique des unités

lexicales : étude de six verbes français

\section{Sentir / sens}

Jean-Jacques Franckel

\section{(2) OpenEdition \\ 1 Journals}

Édition électronique

URL : http://journals.openedition.org/linx/140

DOI : 10.4000/linx.140

ISSN : 2118-9692

Éditeur

Presses universitaires de Paris Nanterre

Édition imprimée

Date de publication : 1 juin 2004

Pagination : 103-134

ISSN : 0246-8743

Référence électronique

Jean-Jacques Franckel, « Sentir / sens », Linx [En ligne], 50 | 2004, mis en ligne le 27 janvier 2011, consulté le 19 avril 2019. URL : http://journals.openedition.org/linx/140 ; DOI : 10.4000/linx.140 


\title{
Sentir / sens
}

\section{Jean-Jacques Franckel \\ Université Paris $X$ - Nanterre \\ UMR 7110 (Université Paris 7)}

\begin{abstract}
Puisque la vérité consiste à ordonner correctement les dénominations employées dans nos affirmations, un homme qui cherche l'exacte vérité doit se rappeler ce que représente chaque dénomination dont il use, et la placer en conséquence : autrement, il se trouvera empêtré dans les mots comme oiseau dans les gluaux; et plus il se débattra, plus il sera englué. C'est pourquoi en géométrie, qui est la seule science que jusqu'ici il ait plu à Dieu d'octroyer à l'humanité, on commence par établir la signification des mots employés, opérations qu'on appelle définitions, et l'on place ces définitions au début du calcul.

Hobbes, Nécessité des définitions, Léviathan, p. 31 Sirey.
\end{abstract}

Cet article ${ }^{1}$ constitue principalement un essai de caractérisation de deux unités lexicales apparentées : sentir et sens. Il s'agira d'appréhender l'identité de ces unités à travers leur variation, en proposant pour chacune une caractérisation en termes de "forme schématique », permettant un raisonnement sur l'organisation de cette variation. Il conduira au passage à examiner, à partir de ce couple d'unités, certains aspects des différences entre les formats de description d'un verbe et d'un nom dans le cadre de cette problématique. Il proposera enfin quelques réflexions sur les relations entre un concept (le concept de «sens») comme enjeu et produit d'une réflexion philosophique, et les propriétés spécifiques du mot par lequel ce concept se trouve incarné dans une langue donnée. On peut considérer que «le sens » n'est rien d'autre, au bout du compte, que le sens du mot sens. Le problème étant que ce mot, comme

${ }^{1}$ Cet article fait suite à deux précédentes tentatives d'analyse du verbe sentir: Lebaud-Franckel (1995), et Franckel (2001), ce dernier étant plus particulièrement pris comme point de départ d'une réflexion sur la notion de glose. Merci aux contributeurs de la revue pour leurs commentaires sur des versions préliminaires de cet article. 
Jean-Jacques Franckel

tous les autres, a non pas un sens mais plusieurs, dont rien ne justifie au demeurant qu'ils puissent être considérés en réalité comme des sens du mot en tant que tel, dans la mesure où ces sens ne se manifestent que dans des interactions avec d'autres mots.

\section{Caractérisation d'une unité en termes de forme schématique}

La caractérisation d'une unité lexicale en termes de forme schématique vise à établir l'identité de cette unité à travers sa variation sémantique. Il s'agit de définir cette identité non par une valeur centrale, dont toutes les autres pourraient être dérivées, mais comme un potentiel dont les différents emplois de l'unité sont autant d'actualisations. Ces actualisations s'effectuent à travers les interactions de l'unité avec les différents types d'environnement que constituent ces emplois.

Le terme d'interaction est à prendre au sens fort. Il est banal de relever d'une part, que le sens d'un mot dépend de son entourage, et, d'autre part, que le sens d'une phrase dépend de son contexte; mais il faut souligner que la réciproque n'en est pas moins vraie, sous des modalités variables: un mot donné dans un tour donné introduit des déterminations, et par conséquent des contraintes fortes sur son co-texte (i.e. les unités lexicales de son entourage); et, d'un autre côté, une phrase donnée détermine étroitement les conditions contextuelles de son interprétation. Cela signifie que le contexte ne se réduit pas à un entourage qui vient la spécifier de l'extérieur, de façon autonome et indépendante: une phrase, ou plus généralement une séquence (toute suite de mots intelligible) engendre les types de contextualisations et de situations avec lesquelles elle est compatible et qui en constituent les conditions d'interprétation.

Établir l'identité d'un mot comme un potentiel, en lui-même « indicible »" (on ne peut l'appréhender, précisément, qu'à travers la diversité de ses actualisations) signifie donc que cette identité ne correspond pas à une valeur parmi d'autres, réputée plus centrale, ou plus fondamentale. Elle correspond au rôle spécifique que l'unité joue dans la construction du sens des énoncés dans lesquels elle est mise en jeu.

\section{Interaction de l'unité avec son environnement}

Prenons par exemple la construction sens de $Y$ : il est facile d'observer la variation du mot dans des séquences telles que : sens de l'olfaction, de l'bumour, d'un mot, de la vie, le sens de la largeur, de la marche. En même temps, on voit qu'à son tour le sens du mot marche varie selon que l'on a le sens de la marche, ou le goût / le plaisir de la marche (à pied). Autre exemple : il s'avère impossible d'avoir : il est dans la largeur. Il faut passer par sens (sens de la largeur). On observe alors qu'une telle séquence ne peut apparaittre qu'avec la préposition dans. On obtient alors : dans le sens de la largeur. Mais le «frayage»

\footnotetext{
${ }^{2}$ Citons la définition que donne Antoine Culioli (2002), p. 27 : «La forme schématique est cette forme abstraite (métalinguistique) qui permet de simuler par le raisonnement ce qui reste, en soi, inaccessible, toujours entr'aperçu à travers le matériau textuel, à la fois obstacle par son apparente solidité qui s'interpose, et trace où se devine le travail d'une intelligence de l'adaptation, du conjectural et du détour. »
} 
contextuel ne s'arrête pas là : une telle séquence ne peut être employée que dans le contexte d'une action finalisée : mettre (passer l'armoire) dans le sens de la largeur.

Voyons encore quelques exemples de cette interaction avec le co-texte et plus largement avec le contexte dans le cas du verbe sentir.

\subsection{Interaction avec le co-texte (environnement textuel, lexical et syntaxique)}

\subsubsection{Niveau lexical}

Prenons le cas d'une séquence comme je sens un (e) X. On peut observer qu'elle introduit sur le terme $\mathrm{X}$ des caractéristiques qui peuvent être tenues comme révélatrices de certaines propriétés du verbe, en liaison avec le tour syntaxique (transitif), et la détermination du terme X (article $u n)$. Dans cette séquence en effet, le verbe sentir convoque une série de termes de façon privilégiée, mais se révèle peu compatible avec d'autres: je sens une bosse, un creux (mais difficilement une table ou une fenêtre $^{3}$ ); je sens une différence (mais difficilement une ressemblance); un point douloureux (mais difficilement un soulagement, qui appellerait - convoquerait - de son côté le verbe ressentir); je sens une odeur de poisson (mais difficilement, ou à certaines conditions très restrictives je sens un poisson), etc.

\subsubsection{Niveau syntaxique}

On peut observer que la distribution des termes précédents, et corrélativement le sens du verbe, se trouvent modifiés en fonction du tour syntaxique (en particulier, reprise du complément, forme pronominale) et du déterminant du complément.

Notons, par exemple, la différence entre je sens le poisson (domaine olfactif) et je le sens, le poisson, (domaine tactile) ${ }^{4}$.

On peut encore comparer $C a$ sent et $C a$ se sent. Alors que ça est a priori susceptible de renvoyer à tout type d'entité, on observe que sentir vient, en l'absence de tout contexte explicite, spécifier ce type d'entité de façon précise et nettement distincte dans les deux cas : ça renvoie à une émanation dans le premier cas, à quelque chose qui est de l'ordre d'une différence dans le second cas.

Enfin, on peut évoquer l'ambiguité de l'interprétation d'une séquence comme Je sens une drôle d'odeur, dans laquelle je peut être interprété comme source d'une odeur ou comme sujet percevant une odeur.

\footnotetext{
${ }^{3}$ Sauf à mobiliser une situation de type colin-maillard. Il s'agit d'un contexte certes bien particulier, mais en même temps révélateur de ce qui s'avère nécessaire au verbe sentir pour que l'on puisse comprendre un tel tour : il faut, en l'occurrence, un contexte de cécité, une situation dans laquelle on se déplace à l'aveuglette. Le point important que ce contexte est déclenché /imposé par le tour je sens une table.

${ }^{4}$ Le passage au domaine tactile provoqué par la reprise de le poisson s'accompagne de la nécessité d'introduire un localisateur de poisson: je le sens, le poisson, dans la bourriche. Nous reviendrons sur ce point. Cet énoncé peut encore renvoyer au domaine gustatif : je découvre le goût du poisson dans un mets.
} 
Jean-Jacques Franckel

\subsection{Interaction avec le contexte}

La forme d'une séquence détermine le type de contexte (situation) qui constitue les conditions de son interprétation.

Ainsi, dans le cas de je sens une bosse, on peut observer que l'interprétation de cette séquence implique que je sois en mouvement (il faut un contexte du type : quand je touche là, quand je passe mon doigt).

De même, dans le cas de je sens le stylo dans ma doublure, il faut du mouvement, je palpe (ou bien mon stylo remue!). Il s'agit là d'une différence radicale avec le verbe voir, par exemple.

Prenons un autre exemple. Si l'on pose à quelqu'un la question Comment te sens$t u$ ? on peut observer une différence sensible avec les questions apparentables Comment vas-tu et Comment te portes-tu? Or il s'avère que la meilleure façon de mettre cette différence en évidence consiste précisément à expliciter le type de contexte qu'appelle chacune de ces trois séquences. On observe que la question Comment te sens-tu? spécifie étroitement la situation qui la rend interprétable. Elle ne peut être que consécutive à un malaise, un accident, une maladie. On peut difficilement croiser quelqu'un dans la rue en lui demandant à brûle-pourpoint Comment te sens-tu? D'un autre côté, Comment te portes-tu ? entrainerait un contexte non moins spécifique, mais tout différent, de médicalisation 5 .

Ces exemples illustrent le fait que la forme même d'une séquence déclenche / construit le type de contexte / situation qui en permet l'interprétation.

Le rapport entre la séquence et son contexte peut donc être défini à la fois comme étant d'ordre «interne» et «externe». Cette appréhension, que l'on peut appeler dynamique, de la contextualisation, à la fois partiellement externe à une suite de mots mais partiellement déclenchée par elle, conduit à une analyse du sens en devenir. Il ne s'agit pas de partir du produit fini (de l'interprétation d'un énoncé) pour voir comment redistribuer des parcelles de sens aux différents composants de cet énoncé, les étapes qui conduisent à cette interprétation n'ont pas à être analysées en référence à cet état final stabilisé ; il s'agit de partir des potentiels liés à des suites de mots (séquences), de les analyser comme déterminant une sorte de frayage dynamique, comme déclenchant les conditions qui, en aval, vont stabiliser cette interprétation.

\section{Mise en œuvre : quelques éléments d'analyse du verbe sentir}

Nous chercherons à définir dans cette perspective l'identité du verbe sentir à travers une forme schématique propre à rendre compte tout à la fois de la singularité et de la régularité de chaque emploi.

\footnotetext{
${ }^{5}$ D'autres différences peuvent être mises en évidence. Ainsi, il ne se porte pas bien est symétrique de il se porte bien, ce qui n'est pas le cas de il ne se sent pas bien (il défaille) vs il se sent bien (il manifeste une harmonie interne).
} 
Sentir / sens

\subsection{Analyse exploratoire préliminaire $d u$ verbe}

Le verbe sentir se trouve empiriquement et lexicographiquement associé de façon privilégiée à deux notions :

- celle de perception;

- celle d'intuition.

La notion de perception, couramment mobilisée ${ }^{6}$ pour définir sentir n'est en fait que partiellement adéquate. On peut en particulier noter que sentir

- exclut le visuel et l'auditif;

- privilégie l'olfactif, le tactile, le gustatif.

L'apparition de telle ou telle de ces valeurs est liée aux termes mis en jeu dans le co-texte, mais aussi de façon plus complexe comme on l'a vu, aux constructions grammaticales dans lesquelles sentir apparait. Rappelons par exemple que :

- Ca sent correspond à l'émanation d'une odeur, ce qui n'est plus a priori le cas avec le tour pronominal Ça se sent.

- Je sens le bébé peut s'interpréter comme je dégage une odeur de bébé, alors que Je le sens, le bébé se voit a priori associé au domaine tactile ou kinesthésique.

- On observe le même type de différence entre Je sens des pieds : je dégage une odeur de pieds, mes pieds dégagent une odeur, et Je sens les pieds (de mon voisin) : j'en ai une perception tactile.

Le lien avec la notion d'intuition peut être analysé a priori comme provenant du fait que sentir se trouve fondamentalement ancré dans une subjectivité. En effet, contrairement au visuel et à l'auditif, qui correspondent à des stimuli objectivables et localisables, l'olfaction et le gustatif sont liés à des modes de perception internes au sujet. Cela est particulièrement vrai de l'olfaction, pour laquelle ce phénomène a été abondamment commenté dans la littérature - philosophique en particulier - sur la perception et sur les sens. Les critères classificatoires classiques font de la vue le sens noble par excellence et de l'olfaction la faculté la plus subjective, intimiste, intériorisée (et le plus souvent aussi la plus archaïque). Il s'agit de plus d'une perception fugace, impalpable, ponctuelle qui ne se manifeste que dans le temps.

Mais on peut en même temps observer que ces considérations sont partiellement en porte-à-faux avec le comportement du verbe sentir : En effet, celui-ci renvoie facilement au tactile, domaine sensoriel qui ne s'inscrit nullement dans le même rapport entre le « subjectif » et « l'objectif » que l'olfactif ou le gustatif.

Pour analyser plus précisément les deux valeurs du verbe précédemment mises en avant, on peut prendre l'exemple de Je le sens, qui peut correspondre à au moins deux types de contextualisation :

1) Je cherche le stylo qui a glissé dans la doublure de ma veste.

\footnotetext{
${ }^{6}$ Pour une analyse fine, cf. en particulier J. Picoche (1986), p 123 sqq et 134 sqq.
} 
Jean-Jacques Franckel

Je le sens active dans ce cas une interprétation de type perception tactile, et implique alors, comme on l'a souligné, que je bouge, que je tâte mes poches, bref, qu'il $y$ ait un mouvement.

2) Dialogue du type

- Pierre ne va pas venir.

- Comment tu le sais?

- Je le sens.

Comment expliciter le sens de sentir dans ces énoncés?

Dans le premier cas, nous avons vu que l'interprétation consistant à associer à sentir le sens de percevoir laisse échapper la spécificité du verbe. De façon générale le recours à une synonymie locale n'a d'intérêt qu'à titre de démarcation: il s'agit précisément de dégager la spécificité de sentir par rapport à percevoir.

Dans le second cas, on peut proposer d'expliciter la signification de je le sens par: $j^{\prime}$ en ai l'intuition, la conviction intime, d'une part, quelque chose me le dit, d'autre part. On peut considérer que c'est la conjonction de ces deux formulations qui est susceptible de constituer une reformulation satisfaisante. Nous prendrons cette conjonction comme piste vers une caractérisation du verbe, dont l'une des propriétés semble être de constituer comme indissociables les deux pans que sa description dans cet exemple fait analytiquement apparaître de façon dissociée.

\subsection{Vers une caractérisation $d u$ verbe sentir}

Reprenons donc les deux éléments d'explicitation de la seconde interprétation de Je le sens (on peut faire l'hypothèse que sentir synthétise ces deux éléments) : j'en ai l'intuition d'un côté et quelque chose me le dit, de l'autre.

D'un côté, quelque chose me le dit signale l'existence, indépendamment de moi, d'un déclencheur de l'état de choses qui s'impose à moi, à savoir que Pierre ne va pas venir: quelque chose (qui m'est externe) déclenche en moi et pour moi la représentation d'un état de choses, consistant en ce fait que Pierre ne va pas venir.

D'un autre côté, l'intuition marque le caractère purement subjectif et non objectivable de cette représentation (de la non-venue de Pierre). Tout en venant de moi, l'idée de cet état de choses s'impose à moi. Elle ne relève pas d'une élaboration active et raisonnée. Je peux chercher à la combattre s'il s'agit d'un mauvais présage, réagir consciemment à son absurdité si elle m'apparaît comme telle, mais la détermination de cette représentation, qui naît pourtant en moi, m'échappe, elle s'inscrit dans une nécessité dont je ne suis pas le maitre.

Prenons un exemple proche : Je sens que je vais me fâcher.

Quelque chose se produit qui déclenche en moi (S) la construction d'une représentation d'un état de choses, à savoir que je vais me fâcher. Ce quelque chose n'est ce qu'il est que pour $\mathbf{S}$, mais en tant que déclencheur de la représentation que $\mathbf{S}$ en a, il existe indépendamment de $\mathbf{S}$, il constitue un événement situé dans l'espace-temps. D’un côté, donc, ce qui déclenche est extérieur à $\mathbf{S}$ et ramené à sa pure existence de déclencheur ; et, de l'autre, ce qui investit ce déclencheur d'un contenu qualitatif, ce qui établit ce dont il s'agit, vient de $\mathbf{S}$, mais en même temps malgré lui, sans qu'il s'agisse d'une élaboration consciemment maitrisée. $\mathbf{S}$ n'est donc ni maittre du 
déclenchement de la représentation qu'il en donne, ni maitre non plus de cette représentation elle-même : il en est le siège, mais non le concepteur.

\subsection{Forme schématique de sentir}

Il ressort de ce qui précède que sentir établit une dissociation entre l'existence de quelque chose d'un côté, et celle d'une représentation que ce quelque chose déclenche et qui s'impose au sujet, d'un autre côté. En soi, la nature de ce quelque chose est donc indéfinissable, si ce n'est comme déclencheur de la représentation qui s'impose au sujet. Celui-ci n'a donc pas accès directement à l'identité de ce qui déclenche la représentation qui s'impose à lui, d'où l'effet d'opacité, voire de cécité observé à plusieurs reprises. Une différence fondamentale avec le verbe voir, réside, par un raccourci très simplificateur, dans le fait que pour voir quelque chose, il faut savoir ce que c'est. En revanche, sentir rend fuyante et opaque l'identité de ce qui est senti. Il établit une coupure entre la représentation que j'ai de quelque chose et ce qu'est le quelque chose qui déclenche la représentation qui s'impose ainsi à moi $^{7}$. Nous proposerons de synthétiser cette caractérisation par la forme schématique suivante :

Sentir marque

1) une double prédication d'existence :

- prédication d'existence de quelque chose $(\mathbf{E})$, « un existant quelque part» donc lié à un site d'existence. sujet $\mathbf{S}$.

- prédication d'existence d'une représentation $\mathbf{P}$ dont le site d'existence est un

2) La spécification par $\mathbf{S}$ de $\mathbf{E}$ comme déclencheur $\mathbf{d}$ de $\mathbf{P}$.

De manière plus « ramassée », cela donne :

Sentir prédique l'existence pour un sujet (S) de quelque chose (E) qu'il spécifie comme le déclencheur (d) d'une représentation (P) dont il devient le site.

Cette proposition peut être assortie de trois remarques :

$1^{\circ}$ Sont donc en jeu quatre paramètres, que l'on peut aisément ramener à trois, $\mathbf{E}$ et $\mathbf{d}$ correspondant à une même entité (quelque chose); $\mathbf{E}$ note un existant en tant qu'il est spécifié par un sujet $\mathbf{S}$ comme déclencheur $\mathbf{d}$ de la représentation dont il devient le site. Nous le noterons donc en fait E-d, et ne procéderons à une décomposition que dans le cadre de l'analyse du plan de variation fondé sur les différents modes possibles de spécification de $\mathbf{E}$ comme $\mathbf{d}$.

$2^{\circ}$ Cette forme schématique signale une double prédication d'existence : d'un côté, prédication d'existence de quelque chose (E-d); de l'autre, prédication d'existence d'une représentation $(\mathbf{P})$ dont $\mathbf{S}$ est le site. En même temps, $\mathbf{S}$ spécifie $\mathbf{E}-\mathbf{d}$ comme le déclencheur $\mathbf{d}$ de cette représentation. Cette double prédication d'existence de $\mathbf{E}$ et de

\footnotetext{
${ }^{7}$ Ce que l'on pourrait résumer par une formule du genre : sentir marque le déclenchement en $\mathbf{S}$ et pour $\mathbf{S}$ d'une représentation de ce qui déclenche cette représentation. Une telle formulation s'avère toutefois trop «nouée» et ne tend pas de façon suffisamment analytique et décomposée les conditions de la variation du verbe telle que la manifeste la diversité de ses emplois.
} 


\section{Jean-Jacques Franckel}

$\mathbf{P}$ est la condition qui permet en même temps et paradoxalement d'établir que l'un n'existe pas sans l'autre. pour $S$.

Représentation signifie ici : ce qu'est / comment est ce qui est déclenché par E-d

Le fait que $\mathbf{S}$ devienne ainsi site d'existence de déterminations qualitatives $\mathbf{P}$ qui s'imposent à lui signifie que ces déterminations ne relèvent pas d'un processus agentif d'élaboration (conscient, intentionnel, rationnel). $\mathbf{S}$ est en quelque sorte passif: il est un simple site d'existence, un support et non un concepteur; ces déterminations s'inscrivent en lui et pour lui, mais ce n'est pas lui qui les construit. Et d'un autre côté, E-d n'est rien d'autre qu'un existant, spécifié comme déclencheur de ces déterminations qualitatives qui s’imposent au sujet. Cela a deux conséquences :

a) on ne peut pas dire que la prédication d'existence de $\mathbf{E}$-d ait un ancrage «objectif», dans la mesure où $\mathbf{E}$ n'existe que comme déclencheur de $\mathbf{P}$, qui, de son côté, n'existe et n'a de statut strictement que pour $\mathbf{S}$;

b) L'identité de E-d en tant que telle demeure, comme nous l'avons déjà observé, inaccessible à travers sentir. En sentant quelque chose, le sujet ne sait pas ce qu'est ce quelque chose du seul fait qu'il le sent. Il peut naturellement le savoir ou en avoir connaissance par ailleurs, par exemple voir ce qu'il sent au moment où il le sent. Le sujet grammatical du verbe peut correspondre d'un côté à $\mathbf{S}$ qui se trouve placé dans ce rapport de cécité ou d'opacité à $\mathbf{E}$-d, et en même temps au sujet énonciateur qui peut établir une correspondance entre E-d et un terme identifié par ailleurs. Nous analyserons les différentes constructions linguistiques correspondant à ce type de cas. Mais le propre de sentir est de ne rendre E-d accessible qu'à travers sa pure fonction de déclencheur des déterminations qualitatives qui s'imposent à $\mathbf{S}$ et qui sont donc fondamentalement et, pourrait-on dire, presque «autistiquement» subjectives. On peut tout au plus dire par inférence que ce qu'est E-d est de l'ordre des déterminations qualitatives qui s'imposent au sujet.

$3^{\circ} \mathrm{Il}$ importe d'accorder une attention particulière au statut du terme quelque chose, que de nombreuses gloses ont mis en œuvre à plusieurs reprises ${ }^{8}$. Quelque chose a permis de donner corps :

a) au paramètre du déclencheur, dans des gloses où il apparaît en position de sujet grammatical : quelque chose me dit que, quelque chose fait que j'ai une représentation de ce dont ce quelque chose est le déclencheur;

b) mais aussi au paramètre de la représentation $\mathbf{P}$ de ce quelque chose: une représentation indéfinissable ou indicible, que le sujet nomme comme quelque chose, autrement dit ce qu'est et comment est quelque chose.

\footnotetext{
${ }^{8}$ On trouve des phénomènes du même ordre avec des verbes qui touchent ou mettent en jeu la notion de "représentation", en particulier le verbe dire. A côté de l'emploi quelque chose me dit que Pierre ne va pas venir, déjà évoqué dans la glose de je le sens, on peut relever l'emploi ça me dit quelque chose (ça me rappelle, évoque quelque chose, ça fait que j'en ai une représentation) ainsi que l'emploi sans complément : un apéritif, şa te dit?
} 
Ainsi, quelque chose apparaît comme le lieu d'une superposition entre ces irréductibles par essence que constituent $\mathbf{E}$-d et $\mathbf{P}$. Ce phénomène est lié au fait que E-d est précisément un terme qui n'a d'autre statut que celui de déclencheur, ce qui entraîne les effets d'opacité ou de cécité observés, en dehors des cas où $\mathbf{d}$ se présente par ailleurs comme la reprise d'un terme identifié. On observe que le complément direct renvoie fondamentalement à la détermination qualitative $\mathbf{P}$.

Nous avons abouti à cette proposition de forme schématique (désormais FS) en centrant essentiellement notre analyse sur un exemple particulier, tout en gardant à l'esprit les données de notre parcours exploratoire. Reste donc à la déployer en la mettant à l'épreuve de l'ensemble des exemples dans leur diversité. Deux tâches essentielles s'attachent à ce déploiement :

a) Établir la correspondance entre les paramètres de la FS et les termes de l'énoncé, dans le cadre d'une forme de syntaxe du verbe, qui fera l'objet du paragraphe 5.

b) Dégager l'organisation de la variation à partir des paramètres de cette FS, prise comme pôle de structuration de cette organisation.

\section{Variations liées aux différents modes de mise en œuvre de la FS}

Nous ramènerons la variation dans la mise en œuvre de cette FS à deux facteurs aux ramifications multiples. Ces facteurs de variation jouent sur les modes de donation, d'articulation et d'instanciation des paramètres de la FS. Ils ne sont ni calqués ni calés sur les distinctions classiques ou empiriquement prégnantes, telles que celle entre intuition et perception, ou entre chacun des cinq sens. Il s'agit de variations portant sur des modes de construction à partir des paramètres de la FS et non sur les résultats interprétatifs de ces constructions. Cela n'empêche pas certains recoupements locaux, mais les déterminations et les fondements en sont profondément différents.

de $\mathbf{P}$

1) Variation des corrélations entre la prédication d'existence de E-d et celle

a) La prédication d'existence de $\mathbf{P}$ en $\mathbf{S}$ peut être consécutive à l'existence première de E-d (problématique possible de la perception : je sens un courant d'air ; quand je passe mon doigt là, je sens (comme) une bosse). De plus, dans ce cas, E-d peut se trouver mis en correspondance avec un terme préconstruit et identifié par ailleurs : $\mathbf{S}$, en sentant ce qu'il sent reconnaît quelque chose qu'il sait par ailleurs exister (qu'il voit par exemple). On est alors dans une problématique de l'adéquation (sur cette partie du tableau, on sent bien les aplats).

b) Ou bien, à l'inverse, sentir peut marquer que, dans la mesure où se trouve prédiquée l'existence de déterminations qualitatives en et pour un sujet (une représentation s'impose à lui), ces déterminations sont déclenchées par un existant E-d qui n'advient à l'existence que de ce fait même (problématique de «l'intuition » : si une représentation s'impose à moi, c'est que quelque chose déclenche cette représentation, « quelque chose me le dit »); 
Jean-Jacques Franckel

c) Enfin, les deux types de prédication d'existence peuvent être indissociables et fondés réciproquement dans leur actualisation solidaire (ça sent).

2) Variation quant au mode de spécification de $\mathbf{E}$ comme d. C'est sur ce plan que se joue la nécessité de décomposer l'entité $\mathbf{E}$-d et que se justifie une notation à deux termes $(\mathbf{E}$ et $\mathbf{d})$.

a) $\mathbf{E}$ acquiert du fait de sentir la fonction de déclencheur par lequel $\mathbf{S}$ le spécifie (je sens une drôle de chose) : tout se passe alors comme si l'on prédiquait de $\mathbf{E}$ la propriété être déclencheur; $\mathbf{S}$ spécifie $\mathbf{E}$ comme $\mathbf{d}$.

b) $\mathbf{S}$ actualise la fonction de déclencheur propre à $\mathbf{E}$. $\mathbf{E}$ est spécifié comme déclencheur d'une représentation qui correspond elle-même à celle d'un déclencheur (stimulus) : une douleur, une odeur, un gồt. Dès lors que la représentation être odeur s'impose à moi et que cette représentation est déclenchée par quelque chose, ce quelque chose est une odeur (il serait difficile d'avoir : ? je sens quelque chose comme une odeur, ou comme un gô̂t, à comparer avec : je sens quelque chose comme une bosse).

c) E n'a de statut que dans son actualisation, qui est aussi celle d'un déclenchement (Ça sent la rose, cette histoire sent l'arnaque).

Nous déployons ci-dessous ces variations dans leur détail.

\subsection{Variation des corrélations entre prédication d'existence de E-d et prédication d'existence de $P$}

La prédication d'existence d'un terme est relative à un site d'existence. Celui-ci est variable pour ce qui concerne $\mathbf{E}-\mathbf{d}$ mais correspond toujours à $\mathbf{S}$ pour ce qui concerne $\mathbf{P}$. Nous distinguerons trois grands cas :

\subsubsection{La prédication d'existence de E-d est première, et la prédication} de P pour S lui est consécutive

Nous établirons deux sous-cas :

- E-d dont est prédiquée l'existence est mis en correspondance avec un terme préconstruit et identifié par ailleurs. Ce premier sous-cas se caractérise par la détermination du complément du verbe : celui-ci est associé à une reprise (on la sent bien, la ciboulette dans la salade), ou à un possessif (je ne sens plus mes jambes), ou bien encore il s'agit d'un nom propre ou générique (ce pianiste sent Chopin comme aucun autre avant lur).

- E-d correspond à un terme qui n'a aucun statut en dehors de cette prédication d'existence. Le complément du verbe apparait avec l'article indéfini (quand je touche là, je sens une bosse). 


\subsubsection{E-d dont est prédiquée l'existence correspond à un terme préconstruit et identifié par ailleurs}

Le quelque chose (E-d) dont sentir prédique l'existence se trouve renvoyer à un terme préconstruit, identifié et localisé par ailleurs.

Cette double construction est relative à $\mathbf{S}$ en tant qu'il sent, d'une part, et à une autre instance de construction, qui peut être en particulier le sujet énonciateur (So), d'autre part. Ainsi, dans ce cas, E-d se réduit d'un côté pour $\mathbf{S}$ à son statut de pur déclencheur de la représentation dont il devient le site ; d'un autre côté, E-d se trouve mis en correspondance avec un terme identifié par le sujet énonciateur So, qui peut alors établir un rapport d'adéquation entre les propriétés de $\mathbf{d}$ en tant qu'identifié et la représentation de $\mathbf{d}$ qui s'impose à $\mathbf{S}$ du point de vue de sentir. Nous rentrons dans le détail de trois sous-cas.

4.1.1.1.1. L'identification de $\mathbf{d}$ passe par une opération de reprise d'un terme préconstruit

Ce cas correspond à un exemple du type: Là, maintenant, je la sens bien, la ciboulette dans la salade. La ciboulette est un terme identifié et situé (dans la salade) indépendamment du fait que sentir marque que quelque chose déclenche une représentation en $\mathbf{S}$ et pour $\mathbf{S}$ comme étant de la ciboulette. Cette double construction aboutit à un rapport d'adéquation entre $\mathbf{E}-\mathbf{d}$ en tant que rapporté à un terme identifié (la ciboulette, identifiée et située), et la représentation qui s'impose à $\mathbf{S}$ du point de vue de sentir (c'est de l'ordre de être ciboulette). Ce qui donne en raccourci : Je sens effectivement, ou enfin, la ciboulette dont je sais qu'elle est dans la salade. Le fait de sentir est de l'ordre d'une capacité : je suis en mesure de sentir.

Non seulement la représentation qui s'impose au sujet n'est pas forcément gustative (elle peut être tactile ou, de façon plus contrainte, olfactive), mais elle n'est pas forcément perceptive à proprement parler, comme l'illustrent des exemples tels que : Qu'il ne soit plus là pour m'aider, je le sens, ou Sur cette partie du tableau, on sent nettement les aplats en question: nulle nécessité de toucher les aplats pour les «sentir»: on en a une « représentation interne », conforme au savoir de leur présence.

\subsection{E-d est rapporté à $\mathbf{S}$ ou à une partie de $\mathbf{S}$}

Ce cas correspond à un exemple du type: Je sens mes jambes. E-d se trouve identifié à mes jambes. Ce type de cas est contraint, car il est nécessaire que mes jambes puissent jouer une fonction de déclencheur de leur représentation comme être mes jambes. Compte tenu de leur statut de parties du corps ${ }^{10}$, cela nécessite un contexte

\footnotetext{
${ }^{9}$ On notera le caractère difficilement interprétable de Je sens la ciboulette sans préconstruction de la ciboulette. Une telle séquence ne serait interprétable qu'en mobilisant la notion d'odeur, qui relève d'un autre cas (cf. ci-dessous en 4.1.3.1.: je dégage une odeur de ciboulette, ou encore (mais plus difficilement) je hume la ciboulette).

${ }^{10} \mathrm{La} \mathrm{FS}$ de sentir postule que le site d'existence de $\mathrm{E}$ est nécessairement disjoint de $\mathbf{S}$ en tant que site d'existence de $\mathbf{P}$. D'où les contraintes observées lorsque se trouve exister par ailleurs une relation entre les deux, telle qu'entre le corps et ses parties.
} 


\section{Jean-Jacques Franckel}

spécifique: je les actionne sur un mode particulier, ou bien elles sont le siège de courbatures douloureuses, etc. La forme négative (je ne sens pas/ plus mes jambes) paraît plus naturelle. Elle implique, là encore, une action des jambes, mais telle que celles-ci ne jouent plus leur rôle de déclencheur de leur représentation comme telles (comme étant mes jambes).

Plus largement, ce cas correspond aux constructions réfléchies : se sentir bien, mal, fatigué. La représentation $\mathbf{P}$ (être bien, mal, fatiguê) est déclenchée par une circonstance particulière qui lui confère une fonction de déclencheur. Comme on l'a observé, Comment te sens-tu? se distingue nettement de questions comme Comment vastu? ou Comment te portes-tu? Je me sens bien, mal, fatigué est circonstanciel : aujourd'bui, quand je suis là, constituent des circonstances déclencheuses de $\mathbf{P}^{11}$. Nous avons affaire ici encore à une problématique de l'adéquation.

\subsubsection{3 $\mathbf{d}$ est un nom propre ou un terme générique}

Il s'agit d'exemples du type : Ce pianiste sent Chopin comme aucun autre interprète.

S'agissant d'un nom propre, le déclencheur E-d (Chopin, ou la musique de Chopin) correspond à un terme identifié, et la représentation que ce pianiste se trouve en avoir entre dans un rapport d'adéquation avec ce qu'est ce déclencheur tel qu'il se trouve identifié par ailleurs.

Cette construction correspond donc à une dissociation de Chopin :

- d'un côté, Chopin (ou la musique de Chopin, terme identifié) a un mode d'existence propre et indépendant de $\mathbf{S}$. E-d résulte de la prédication d'existence de quelque chose correspondant à Chopin (lorsque ce pianiste en interprète la musique).

- d'autre part, (être) Chopin est une représentation purement subjective qui s'impose à $\mathbf{S}$, et dont il est le site.

Dans ce cas, interviennent deux facteurs supplémentaires :

- la prédication d'existence de $\mathbf{E}-\mathbf{d}$ et de $\mathbf{P}$ est directement liée au processus d'interprétation de la musique de Chopin par ce pianiste. D’un coté, cette interprétation fait de la musique un déclencheur, de l'autre elle manifeste la représentation (être Chopin) qui s'impose à $\mathbf{S}$;

- c'est l'énonciateur So qui juge idéale cette manifestation relativement à ce qu'est pour lui la musique de Chopin.

Résumons les caractéristiques essentielles de ce premier cas, avec ses trois sous-cas :

- E-d est premier: c'est dans la mesure où E-d fait l'objet d'une prédication d'existence que $\mathbf{S}$ est le siège d'une représentation.

\footnotetext{
${ }^{11}$ Le cas de se sentir bien dans sa peau est différent, n'étant plus (nécessairement) de l'ordre d'un prédicat subjectif (cf. on le sent bien/mal dans sa peau).
} 
- E-d correspond à un terme identifié par ailleurs (il y a quelque chose, et ce quelque chose correspond à un terme identifié). Il s'ensuit que la représentation qui s'impose à $\mathbf{S}$ peut être considérée comme une représentation de ce qu'est $\mathbf{E}-\mathbf{d}$ (lorsque je sens la ciboulette, je peux savoir qu'il s'agit de ciboulette). Cela n'est nullement le cas dans d'autres emplois que nous allons examiner, où la représentation $\mathbf{P}$ demeure dans une opacité relativement à l'identité de $\mathbf{E}$-d.

- E-d, en tant qu'identifié indépendamment de sa fonction de déclencheur peut entrer dans un rapport d'adéquation avec la représentation que $\mathbf{S}$ en a. Ce rapport d'adéquation se trouve d'ailleurs massivement marqué par des adverbes d'adéquation: $\mathbf{S}$ sent nettement, plus ou moins distinctement, idéalement, de façon harmonieuse ou discordante, etc.

4.1.1.2. E-d n'a aucun statut en dehors de sa prédication d'existence. Le complément du verbe sentir apparaît avec l'article indéfini (un)

La présence de l'article un devant le complément constitue la trace d'une prédication d'existence. Celle-ci nécessite un site d'existence qui peut prendre différentes formes.

4.1.1.2.1. Construction en bloc de l'existence de $\mathbf{E}$-d et de son site d'existence

Le site d'existence de E-d est donné en même temps qu'est prédiquée cette existence. Ce cas peut être illustrée par l'exemple Quand je touche là, je sens une bosse. E-d et son site d'existence (là où je touche) sont donnés à travers un mouvement de $\mathrm{S}$. La fonction de déclencheur par laquelle $\mathbf{S}$ spécifie $\mathbf{E}-\mathbf{d}$ correspond au fait que le site d'existence de $\mathbf{E}$-d est en même temps ce par rapport à quoi il se distingue (la surface lisse) et acquiert ainsi cette fonction de déclencheur : E-d est un point singulier qui est en même temps partie prenante de la surface lisse qui constitue son site d'existence.

Il y a localisation pour $\mathbf{S}$, relativement à un site donné, de quelque chose qui, relativement à ce site, prend le statut de déclencheur d'une représentation : il s'agit d'un point singulier qui, tout en étant localisé par ce site, s'en distingue, prend le statut d'une différence, non identifiable autrement que par ce caractère de différence. Le site et ce point singulier sont construits dans le même mouvement: la construction du point singulier est indissociable de celle du fond dont il se détache.

On pourrait être tenté, sans les détours de l'analyse proposée, de paraphraser cet exemple de la façon suivante : il y a une bosse et je la perçois. Une telle formulation apparaît toutefois doublement erronée du point de vue de l'analyse qui sert de fil conducteur, et qui vise à considérer chaque exemple comme relevant d'un type de variation parmi d'autres de la mise en œuvre de la forme schématique. En effet, Je sens une bosse ne constitue pas une prédication d'existence d'une bosse (il y a une bosse est donc une glose inadéquate). Il y a bel et bien prédication d'existence de quelque chose (marquée par l'article un), mais sentir dissocie la prédication d'existence de ce quelque chose de sa représentation comme être bosse. La glose serait donc bien plutôt de ce point de vue : il y a quelque chose $(\boldsymbol{E})$ dont la spécification que je peux en donner se réduit au fait qu'elle déclenche en moi une représentation de bosse $(\boldsymbol{P})$. 
Jean-Jacques Franckel

4.1.1.2.2. S introduit ou constitue le site d'existence de $\mathbf{E}-\mathbf{d}$

Il s'agit ici d'exemples où $\mathbf{S}$, site de $\mathbf{P}$, coïncide avec le site d'existence de $\mathbf{E}$-d. Je sens une douleur, un courant d'air, une drôle de chose.

\subsection{Le cas de une odeur}

Une odeur présente la propriété d'être auto-localisée, elle constitue son propre site d'existence : c'est une émanation, elle est flottante, fugace, évanescente, se trouve localisée là où elle se trouve, elle constitue à elle-même son propre site d'existence. Nous réservons ce commentaire aux seuls exemples où le terme même d'odeur est mobilisé, comme dans je sens une odeur de rose, où une odeur correspond à un déclencheur posé comme identifiable mais non identifié et être rose la représentation que le sujet se trouve en avoir. L'exemple ça sent la rose relevant d'un autre cas de figure.

Une caractéristique de l'exemple: je sens une odeur est que l'odeur est, explicitement ou non qualifiée (étrange, indéfinissable, tendanciellement désagréable). Nous proposerons une explication de ce phénomène dans le cadre du deuxième ordre de variation (4.2.2.).

\subsubsection{La prédication d'existence de $P$ pour $S$ est première, et la} prédication de E-d en est une conséquence

Dans la mesure où se trouve prédiquée l'existence de déterminations qualitatives en et pour un sujet (une représentation s'impose à lui), il s'ensuit que ces déterminations sont déclenchées par un existant E-d (problématique de «l'intuition»). La prédication d'existence de E-d est entièrement subordonnée à la prédication d'existence de $\mathbf{P}$ en et pour $\mathbf{S}$. C'est la « représentation qui fait l'existence ».

\subsubsection{Intuition}

Ce cas renvoie en particulier aux valeurs de type «intuition». Une représentation s'impose au sujet et se trouve secondairement spécifiée comme ayant pour source un déclencheur $\mathbf{d}$ qui a un statut indépendamment de cette représentation. L'existence d'un déclencheur est une conséquence de la représentation telle qu'elle s'impose au sujet. Ce cas peut être illustré de façon exemplaire par le dialogue précédemment commenté : Comment tu le sais? Je le sens, ou encore Ce genre de choses, c'est inexplicable, ça se sent, c'est de l'ordre de l'intuition. Étant donnée une représentation d'un état de choses, sentir marque que celle-ci est déclenchée par une source externe (d'où la requalification d'un savoir, ou d'une certitude) mais qui n'a d'existence qu'à travers celle de cette représentation même.

\subsubsection{E-d n'a d'existence qu'à travers l'actualisation d'une représentation}

$\mathbf{P}$ correspond à un processus, c'est une représentation qui advient à l'existence sous la forme d'un événement. Le complément de sentir se présente sous la forme d'un verbe à l'infinitif, ou d'une complétive. Le déclencheur de cette représentation est 
alors événementiel, et $\mathbf{P}$ est lui-même processif et associé à un prédicat: Je sens que je vais me fâcher. La représentation du processus se fâcher s'impose à moi, et il est posé qu'elle est déclenchée par un phénomène dont rien d'autre ne peut être dit que le fait même qu'il déclenche cette représentation. Ce cas correspond encore à la vaste cohorte des exemples du type je te sens venir, je sens la lassitude me gagner, l'angoisse m'étreindre, la colère m'envabir, le doute m'oppresser, un frisson me parcourir des pieds à la tête, un ennui profond s'installer en moi, etc.

\subsubsection{Les deux prédications d'existence sont contruites en bloc et fondées solidairement dans leur actualisation}

Dans le premier cas (4.1.1.), l'existence du terme qui prend la fonction de E-d est fondée indépendamment de sentir, elle est première. Dans le deuxième cas (4.1.2), l'existence de $\mathbf{E}-\mathbf{d}$ n'est fondée que du fait même que $\mathbf{S}$ se trouve être le siège d'une représentation. On peut alors dire que c'est la représentation qui fonde l'existence de ce qui la déclenche.

Dans ce troisième cas, l'existence de $\mathbf{E}-\mathbf{d}$ et $\mathbf{P}$ est fondée réciproquement et en bloc : E-d et $\mathbf{P}$ n'ont d'existence et de statut que l'un par l'autre.

\subsubsection{Cette table sent la cire}

Dans cet exemple, il y a processus de déclenchement, dans lequel le déclencheur n'est ni identifié, ni identifiable. Le déclenchement détermine en bloc le déclencheur et la représentation qui s'impose au sujet comme être cire. La table ne correspond pas directement au déclencheur en question. La table localise quelque chose $(\mathbf{E})$ dont tout ce que le sujet (correspondant ici à l'énonciateur) peut dire est qu'il déclenche en lui une représentation être cire. C'est le lieu où et par lequel s'actualise le déclenchement d'une représentation.

Dans ce type d'exemple, l'existence du déclencheur n'est donc pas identifiable, contrairement à l'exemple pourtant voisin en apparence : je sens une odeur de cire (où se trouve dissocié une odeur comme déclencheur et sa représentation comme être cire). Dans le cas de cette table sent la cire, rien n'indique la présence de quoi que ce soit d'identifiable. La propriété être cire n'a de statut que par la représentation déclenchée chez le sujet. De même, dans un exemple comme cette histoire sent l'arnaque, l'arnaque n'est présente que par la représentation qui s'impose au sujet, en relation avec cette bistoire qui fait que ça sent l'arnaque : quelque chose fait que j'ai une représentation de l'ordre de l'arnaque, eu égard à cette histoire qui fait qu'il y a déclenchement de cette représentation. Du même coup, le sujet syntaxique, à travers sa fonction factitive, hérite de la propriété d'être à la source du processus que marque sentir: la table est odoriférante; l'affaire est véreuse.

\subsubsection{L'article le dans sentir le...}

Pour analyser ces exemples, il convient de partir de l'observation de deux propriétés fondamentales des compléments de sentir dans ce type de cas : 
Jean-Jacques Franckel

$1^{\circ} \mathrm{Ils}$ sont nécessairement introduits par l'article le (ce qui n'est pas nécessairement le cas dans les exemples analysés ci-dessus, où le terme même d'odeur est mobilisé, comme je sens une odeur de rose). On aurait difficilement je sens une cire, un poisson ou un fromage sauf à passer à une interprétation tactile qui, comme nous l'avons vu précédemment impliquerait un mouvement exploratoire du sujet qui introduit alors le site d'existence de $\mathbf{d}$ (quand je touche là....).

L'article le pointe ce qui vérifie la propriété être $X$ dans une classe d'entités hétérogènes. L'article le confère donc une indépendance à la propriété par rapport à la prédication d'existence du terme qui en constitue le support. On part d'une propriété singulière et l'on désigne dans une classe représentée contextuellement ce qui relève de cette propriété singulière : le qui est $X$ dans la classe des objets environnants. La propriété est attribuée indépendamment de la construction du terme auquel elle est rapportée.

De ce point de vue, il existe une affinité entre la dissociation entité / propriété marquée par le et la dissociation $\mathbf{d} / \mathbf{P}$ qu'établit sentir.

$2^{\circ}$ Ils mobilisent de façon privilégiée (mais nullement exclusive) des termes qui sont sémantiquement compatibles avec la propriété être odeur. Un exemple comme je sens l'air relèverait d'un autre cas : il ne prend pas a priori une interprétation olfactive et implique un prédicat: l'air qui passe, l'air qui me pénètre. En revanche je sens l'essence prendra plutôt une interprétation olfactive en raison des propriétés volatiles de l'essence. Dans le cas de le poisson, c'est sentir qui pose une dissociation entre l'actualisation d'un déclencheur, qui en soi n'a d'autre propriété que d'être un déclencheur, et les propriétés dont l'investit un sujet. L'interprétation comme odeur de je sens le poisson provient du fait que le poisson n'a de statut que dans l'actualisation de son déclencheur. Poisson ou fromage ne se définissent pas intrinsèquement comme dotés d'odeur ${ }^{12}$, mais deviennent interprétables comme tels dès qu'ils se trouvent associés au verbe sentir sans mention d'un site de localisation. Lorsque ces termes font par ailleurs l'objet d'une localisation, l'interprétation comme odeur disparait (cf. la différence entre je sens le poisson et je sens le poisson dans la bourriche).

\subsubsection{3. Ça sent}

L'interprétation «olfactive » de la séquence Ça sent répond au même principe. Cet exemple, qui par son minimalisme permet d'atteindre sous un angle particulier la quintessence du verbe, n’introduit ni site localisé ni $\mathbf{P}$ spécifié. Cet exemple constitue l'illustration emblématique du fait que dans ce type de cas, le déclencheur consiste en

\footnotetext{
${ }^{12}$ Le fait que quelque chose sente la vanille n'implique nullement présence ou existence de vanille. La vanille telle que la détermine sentir correspond à la propriété être vanille et non une propriété de son déclencheur (puisque le déclencheur n'a intrinsèquement aucune propriété en dehors de celle de déclencheur). On sait par ailleurs que la dénomination des odeurs passe par celle de leur source. La dénomination de l'odeur par celle de sa source n'implique nullement que l'existence de l'une (pour un sujet) soit indissociable de la présence de l'autre. Remarquons enfin que l'on peut chercher à établir le nom d'une odeur (dans certains cas d'un goût) - ce qui implique de passer par la dénomination de sa source - mais non d'un bruit ou d'une vue. Le nom d'une vue est directement celui de ce qui est vu (Sur ce point, cf. notamment J. Fontanille, 1999).
} 
sa propre actualisation (déclenchement fugace, éphémère, dans l'instant), $\mathbf{P}$ se réduisant de son côté au simple fait de l'existence de ce qui n'est construit que dans l'instant, dans une pure actualisation. On peut illustrer cette analyse en redéployant Ça sent sous la forme $C$ a sent quelque chose (bon ou mauvais) ${ }^{13}$.

Le déclencheur comme la représentation n'ont de statut que dans leur solidaire actualisation.

Ce cas est donc très différent de ça se sent, qui relèverait du cas précédent 4.1.1. Dans cet exemple, ça est alors de l'ordre d'une différence. Or une différence implique l'existence de deux entités ou de deux sites entre lesquels elle s'établit.

\subsubsection{Synthèse : reformulation de ce plan de variation en termes d'adéquation, d'attribution d'une propriété et d'actualisation}

Les trois grands cas inscrits dans ce plan de variation peuvent être reformulés respectivement en termes 1) d'adéquation (un rapport de plus ou moins grande félicité est établi entre ce qu'est un terme et la façon dont il est senti) ; 2) d'attribution d'une propriété à un terme (un terme devient le support de la représentation qu'il déclenche, et hérite de cette propriété de déclencheur), 3) d'actualisation (privilège de l'odeur dans son évanescence).

Ces trois cas peuvent être associés aux trois grands types de construction des occurrences d'une notion, appelés dans ce cadre général : discret, compact et dense $e^{14}$.

\subsection{Modes de spécification de E commed}

Ce second plan de variation concerne le mode de construction d'un existant en tant que déclencheur $\mathbf{d}$. Il implique donc de dissocier $\mathbf{E}$ et $\mathbf{d}$. Nous distinguerons trois cas.

\subsubsection{Un existant acquiert la fonction de déclencheur}

Un existant acquiert la fonction de déclencheur du fait que $\mathbf{S}$ le spécifie comme tel. L'existant est «quelque chose » auquel est attribuée la fonction de déclencheur, comme dans l'exemple : Je sens une bosse, une drôle de chose, un truc bizarre.

Il y a un existant dont est prédiquée la propriété de déclencher la représentation que $\mathbf{S}$ se trouve avoir. Une représentation se manifeste à $\mathbf{S}$ à partir d'un existant qui prend du coup la fonction de déclencheur de cette représentation : je sens une bosse peut être, de ce point de vue, glosé par : il y a quelque chose à quoi je confere la propriété de déclencher la représentation que j'en ai d'être bosse,

On peut rejouer les variations du premier plan. Par exemple, E-d peut être caractérisé par l'opacité qui s'attache à la représentation $\mathbf{P}$ : la glose précédente établit

\footnotetext{
13 L'effet est donc différent de celui d'un exemple comme je sens une odeur, où l'odeur tend à être désagréable, ou du moins bizarre, comme nous le verrons au $\ 4.2 .2$.

${ }^{14}$ Sur ces notions, cf. en particulier S. de Vogüé (1999). Au sein des trois grands cas distingués (4.1. à 4.3) ces modes de construction se rejouent dans différents sous-cas. Ainsi dans le cas 4.1., la ciboulette est sentie (discret) ; en sentant Chopin l'interprète devient un «Chopinien » (compact); il y a perception actualisée de mes jambes (dense).
} 
Jean-Jacques Franckel

que $\mathbf{S}$ a une représentation non pas directement d'un existant, mais d'une propriété de cet existant qui est d'être un déclencheur : quelque chose de l'ordre de, quelque chose comme. Je sens quelque chose dont je ne sais pas exactement ce que c'est, mais dont je sens que cela déclenche la représentation que j'ai comme déclencheur sous la qualité être bosse, être douleur. Mais E-d peut aussi être rapporté à un terme identifié comme dans l'exemple : je sens mes jambes. Ce qui fonde ici $\mathbf{E}$ comme déclencheur, tout comme dans le cas de bosse, est qu'il fait l'objet d'une prédication d'existence à travers un mouvement: il faut que j'actionne mes jambes pour que quelque chose relatif à mes jambes déclenche la représentation être mes jambes qui s'impose à moi ; de même il faut qu'en palpant une surface lisse un contraste apparaisse, pour que le quelque chose qui déclenche ma représentation être bosse soit spécifié comme déclencheur.

\subsubsection{S actualise la fonction de déclencheur propre à $E$ : la représentation qu'il déclenche est de l'ordre de être déclencheur}

Dans le cas précédent, tout se passait comme si l'on prédiquait de $\mathbf{E}$ la propriété être déclencheur. Ici, $\mathbf{E}$ est un déclencheur, il a les propriétés intrinsèques d'un stimulus. Lorsque je sens une odeur, la représentation être odeur s'impose à moi, et dès lors que je spécifie quelque chose $\mathbf{E}$ comme déclencheur de cette odeur, il s'agit d'une odeur. Alors que toutes sortes de choses peuvent déclencher une représentation en termes de être bosse (cf. je sens quelque chose comme une bosse), seul un goût déclenche une représentation être goût. Cette coïncidence entre $\mathbf{E}$ et $\mathbf{P}$ que sentir pose comme disjoints par construction se solde par une contrainte immédiate: l'odeur ou le goût en question doivent être qualifiés. En l'absence de qualification explicite, l'odeur ou le goût s'avère de quelque façon anormal et porteur d'une bizarrerie qui pérennise l'opacité dans la coïncidence.

Il y a donc adéquation entre $\mathbf{P}$ qui est de l'ordre de être un stimulus, et $\mathbf{E}$ qui se trouve spécifié comme déclenchant $\mathbf{P}$.

Nous distinguerons ici deux sous-cas : 1) le déclencheur est un stimulus externe (odeur, goût) ; 2) le déclencheur est un stimulus interne (frisson, angoisse).

\subsubsection{Le déclencheur est un stimulus externe}

Il s'agit d'exemples du type ça sent une drôle d'odeur; ce plat sent une odeur bizarre ; je sens un goût de rance; je sens un courant d'air. Dans ces exemples, une odeur ou un goût est ramené à sa fonction de déclencheur de quelque chose d'étrange en matière d'odeur ou de goût. L'existant (une odeur, un gô̂t) est en tant que tel un déclencheur. Ce n'est pas sentir qui pose quelque chose comme déclenchant: ce quelque chose est un déclencheur, et sentir marque que $\mathbf{S}$ se trouve en avoir une représentation, qui prend la forme d'une qualification de ce déclencheur.

Ce qui est senti n'est pas de l'ordre d'une odeur ou d'un goût, ni d'un courant d'air: c'est une odeur ou un goût, ou un courant d'air qui se trouve déclencher sa représentation sous telle ou telle qualification. Le critère décisif mis en avant est que le caractère subjectif de la représentation qui s'impose tient non pas à la nature de l'existant (une odeur, un goût, etc.) mais à sa qualification. L'opacité change d'objet: elle ne réside plus dans la nature du déclencheur, mais dans le fait que la représentation de 
S est indissociable de l'incapacité de l'identifier précisément. D'où le fait que l'odeur (ou le goût) tend à être désagréable: non reconnaissable, non immédiatement rapportable à du connu.

\subsubsection{Le déclencheur est interne}

Dans l'exemple Je sens un frisson me parcourir des pieds à la tête ou je sens l'angoisse m'étreindre, le déclencheur n'est pas de l'ordre de être frisson ou être angoisse. En tant que spécifié comme déclencheur de être frisson ou être angoisse, le déclencheur est un frisson ou une angoisse. On se trouve dans un cas où la coupure propre à sentir entre $\mathbf{P}$ et $\mathbf{E}-\mathbf{d}$ ne se solde pas par une opacité, du fait que l'un et l'autre sont internes au sujet.

\subsubsection{Construction dans une actualisation solidaire d'un existant et de sa fonction de déclencheur}

L'existant n'est construit que dans son actualisation, il est de l'ordre d'un événement, et cet événement même est celui d'un déclenchement, par lequel ne peuvent être dissociés un existant de l'actualisation du déclenchement.

On retrouve, mais sous un autre angle, l'exemple cette table sent la cire. Il y a quelque chose qui fait que (en relation à cette table) se trouve déclenchée la représentation être cire. Or ce quelque chose n'est autre que l'événement même de ce déclenchement. Il n'y a d'existant-déclencheur que dans la stricte mesure où il y a déclenchement ${ }^{15}$.

\section{Questions de syntaxe}

\subsection{Mise en correspondance des paramètres de la forme schématique avec des termes de l'énoncé}

L'analyse des différents emplois de sentir en termes de forme schématique doit, comme pour tout verbe (et pour toute unité), passer par un travail de mise en correspondance systématique des paramètres de cette forme schématique avec des termes qui se trouvent ou non matérialisés dans l'énoncé, et qui, lorsqu'ils le sont, peuvent occuper des places d'argument variables ${ }^{16}$.

\footnotetext{
${ }^{15}$ On retrouve dans ce plan de variation les trois grands cas ébauchés précédemment: respectivement compact (la propriété de déclencheur est attribuée à un existant); discret (un existant, odeur ou goût, joue bien le rôle de déclencheur qui lui est propre); dense (il n'y a d'existantdéclencheur que dans la stricte mesure où il y a déclenchement). L'exemple cette table sent la cire peut être analysé de plusieurs points de vue, qui montrent que les constructions se rejouent selon le point de vue considéré. La table acquiert la propriété (pour faire bref) d'être odoriférante (compact). Mais l'existence de $\mathrm{d}$ et $\mathrm{P}$ est fondée réciproquement dans leur actualisation, en même temps qu'il n'y a d'existant-déclencheur que dans la stricte mesure où il y a déclenchement (dense).

16 On peut faire l'hypothèse que cette mise en correspondance répond, au delà de chaque cas particulier à des règles d'organisation générales relevant d'une forme de syntaxe qui n'aurait alors plus rien d'argumentale. La mise au jour de telles règles implique la prise en compte du plus grand nombre possible de verbes analysés dans le format du présent numéro de LINX.
} 
Jean-Jacques Franckel

\subsection{La question de la transitivité et du statut du complément}

Le verbe sentir peut être caractérisé comme fondamentalement transitif, si du moins l'on se donne pour critère la présence d'un complément direct $\mathrm{C}_{1}$. Il existe toutefois des emplois sans complément : ça sent. La nature du complément, ainsi que son absence possible correspondent à des cas de dissociation entre le fait même que le sujet devienne siège d'une représentation déclenchée par un élément $\mathbf{d}$ et la spécification de cette représentation.

\subsection{1. Ça sent!}

Le «degré zéro » de la détermination qualitative de $\mathbf{P}$ correspond à la forme sans complément, que l'on trouve dans ça sent! Dans cet énoncé (dont la forme détermine une intonation de type exclamatif), les paramètres de la forme schématique ne font l'objet d'aucune matérialisation. Nous proposons de poser que $\mathbf{P}$ peut être rendu par quelque chose, encore qu'une différence doive être faite entre ça sent et ça sent quelque chose, dont on pourrait dire qu'il représente non pas un degré zéro, mais un degré minimal, et explicité comme tel, de spécification. L'absence de toute spécification, de même que la spécification la plus indéterminée marquée par quelque chose renvoie au fait que la représentation $\mathbf{P}$ se réduit ici au fait même qu'elle se trouve déclenchée par un déclencheur: $\mathbf{S}$ se représente le fait que quelque chose déclenche ce fait (le fait ne vient pas de So, et So devient siège de la représentation de l'existence de ce fait). La présence de quelque chose ajoute que So ne peut rien en dire d'autre. Rappelons (4.1.3.3.) qu'en l'absence de tout déclencheur identifié ou même identifiable, celui-ci n'a d'autre statut que sa propre actualisation.

\subsubsection{Complément introduit par un}

Dans le cas où le complément est introduit par l'article un, deux cas peuvent être distingués :

- les exemples du type : une bosse. Nous avons montré que dans ce cas, une bosse peut être analysée comme une spécification du quelque chose que je sens (quand je touche, par différenciation de la surface lisse dont elle émerge), bosse pouvant être décrit comme quelque chose de protubérant. Rappelons ici les contraintes sur ce type de spécification : des séquences comme je sens une voiture, une maison, etc. sont des plus contraintes. Le quelque chose auquel renvoie $\mathbf{d}$ est de l'ordre de être bosse.

- les exemples du type je sens une odeur (de rose). Cette fois, odeur n'est pas la spécification du quelque chose que je sens, c'est directement la représentation qui s'impose à moi du déclencheur de cette représentation ${ }^{17}$ : c'est une odeur que je spécifie par une qualification (cf. 4.2.2). En l'absence de tout adjectif explicite, on a vu que l'odeur est précisément spécifiée comme non spécifiable et par là même bizarre, anormale, facilement désagréable. Dans ces exemples, le complément

\footnotetext{
${ }^{17}$ Il s'agit toujours d'un « stimulus » : une odeur, une douleur, un courant d'air (cf. 4.2.2.).
} 
correspond au déclencheur, et sa qualification à la spécification qu’il déclenche. Le quelque chose auquel renvoie $\mathbf{d}$ est une odeur.

Dans un exemple comme je sens une différence, différence correspond à la spécification de ce qui se présente comme le déclencheur potentiel d'une différence, à savoir une comparaison. Le fait de comparer est le déclencheur de la spécification de la comparaison comme différence.

\subsubsection{Complément introduit par le}

Dans le cas où le complément est introduit par l'article le, le $\mathrm{N}$ renvoie à la représentation d'un terme identifié par ailleurs (je sens nettement la ciboulette dans la salade), ou non (ça sent la cire, l'arnaque). Dans ce cas, décrit comme discret, qui renvoie à une problématique de l'adéquation, nous avons vu que ce terme prend alors ici encore un double statut. D'un côté il correspond à $\mathbf{d}$ (quelque chose que l'énonciateur sait être de la ciboulette (celle qu'il a mise lui-même, par exemple); de l'autre, il correspond à $\mathbf{P}$ (spécification de $\mathbf{d}$ comme être ciboulette, en tant qu'il la sent).

Dans l'exemple ce pianiste sent Chopin comme aucun autre interprète, Chopin fait l'objet du même type de dédoublement : il correspond à un télescopage entre $\mathbf{d}$ et $\mathbf{P}$.

\subsection{Le statut du sujet grammatical}

Le sujet grammatical $\mathrm{C}_{\mathrm{o}}$ peut soit correspondre à $\mathbf{S}$, soit ne correspondre à aucun paramètre de la forme schématique. Cela signifie en particulier que $C_{o}$ ne correspond jamais à $\mathbf{d}$.

\subsubsection{Co correspond à $S$}

Il s'agit des exemples du type je sens que je vais me fâcher; je sens une drôle d'odeur (dans cette pièce); il sent Chopin comme personne.

Comme on l'a vu, $\mathbf{S}$ peut être pris dans un rapport à l'énonciateur (So). Ainsi, un exemple comme tu sens cette odeur? où tu correspond à $\mathbf{S}$ renvoie à une prise à témoin du fait que l'énonciateur So sent cette odeur. Tu correspond, dans le cadre de la question posée, à $\mathbf{S}$ qui, par ailleurs, se trouve renvoyer en même temps à So (l'énonciateur d'une telle question sent une odeur).

\subsubsection{Co ne correspond à aucun des paramètres de la forme schématique}

Dans un exemple comme cette table sent la cire, nous avons argumenté (4.1.3.1.) l'analyse selon laquelle cette table ne correspond pas directement au déclencheur d. La table a la valeur d'un factitif: ce qui fait que le processus de sentir a lieu, c'est le lieu où et par lequel il y a déclenchement d'une représentation en termes de être cire.

C'est de ce double statut possible du sujet grammatical que provient l'ambivalence, souvent mise en évidence, de je sens une odeur bizarre, où je peut soit ne correspondre qu'à $\mathbf{S}$ (ici identifié au sujet énonciateur), soit être aussi posé comme terme relativement auquel le déclenchement se produit : ça sent le poisson, et je mets cela en relation avec moi. Je prend alors le même statut factitif que la table: je suis déclencheur du 
Jean-Jacques Franckel

fait que ça sent une drôle d'odeur, ou encore je suis déclencheur de la présence d'une odeur qui déclenche sa spécification comme odeur bizarre.

\subsection{Constructions syntaxiques complexes}

Sentir peut entrer dans une série de constructions syntaxiques, en particulier pronominales et prépositionnelles. Nous avons eu l'occasion d'évoquer des exemples du type je me sens bien. Mais il existe des constructions plus complexes, que nous ne ferons que mentionner ici, telles que il ne se sent plus qui implique la forme négative, et des constructions prépositionnelles telles que se sentir de faire quelque chose qui implique la forme négative (Je ne me sens pas d'y aller tout seul), ou interrogative (Est-ce que tu te sens d'y aller tout seul ?), ou encore il ne se sent plus de joie. L'analyse de ces constructions impliquerait la prise en compte des propriétés des éléments constitutifs du tour, et en particulier de la préposition de et dépasserait le cadre de cet article. Du point de vue du fonctionnement de sentir, ces exemples renvoient au cas d'adéquation (4.1.1.1). Pour faire bref : il ne se sent plus marque qu'il n'y a plus d'adéquation entre ce qu'il est d'un côté, et la représentation être lui telle qu'elle s'impose à lui et telle qu'il la manifeste.

\section{Du verbe sentir au nominal sens}

Nous défendrons l'hypothèse selon laquelle la forme schématique de sens met en jeu, mais sous une autre configuration, les mêmes paramètres $(\mathbf{S}, \mathbf{d}$ et $\mathbf{P})$ que celle du verbe sentir. Le fondement de cette hypothèse passe par un parcours descriptif des emplois de sens.

\subsection{Parcours descriptif des emplois de sens}

Nous distinguerons dans une première phase descriptive (avant de les analyser comme déploiements de la forme schématique qui sera proposée) deux types d'emplois de sens : 1) les emplois absolus ; 2) les emplois prépositionnels (sens de Y).

\subsubsection{Emplois absolus de sens}

Ils sont de plusieurs types :

\section{sensoriels ") \\ 6.1.1.1. Au pluriel: l'empire des sens, les cinq sens (« récepteurs}

On peut analyser sens comme ce qui permet à un stimulus (qu'on appellera donc à nouveau $\mathbf{d}$, pour déclencheur) de rendre $\mathbf{S}$ capable de construire pour lui-même et de s'approprier (sur tel ou tel mode «sensoriel», olfactif, auditif, etc.) les déterminations du contenu qualitatif $(\mathbf{P})$ de ce quelque chose : ce qu'est / comment est / en quoi consiste ce quelque chose. La formulation de cette description constitue une première illustration de la résurgence des paramètres de la forme schématique de sentir. 


\subsubsection{Emplois de sens comme concept philosophique ou religieux}

Il s'agit d'exemples comme qu'est-ce que le sens? la quête / la crise du sens. Sens tend dans ce cas à être associé à une dimension téléologique, et n'est donc pas sans parenté avec les cas où il prend une valeur proche de direction, orientation vers un but (cf. cidessous 6.1.1.6.).

\subsubsection{Emplois où sens s'apparente à signification / interprétation, et relève d'un vouloir dire}

On obtient cette valeur dans des tours tels que en ce sens (à distinguer de dans ce sens qui renvoie aussi possiblement à une direction), ou encore sens à donner à (quel sens donner à cet événement? à ce comportement? etc.). Sens est alors associable à un vouloir dire.

\subsubsection{Emplois dans des expressions du type : tomber sous le sens, avoir $d u$ sens, ou en association possible avec l'adjectif bon ou commun}

À côté de avoir du sens qui est le prédicat de quelque chose, on observe que avoir $d u$ bon sens peut être aussi le prédicat d'un sujet. Plus précisément, il s'avère que avoir $d u$ sens n'apparait de façon immédiatement naturelle que dans des emplois du type : cela n'a pas de sens, ou encore quel sens ça a? qui présentent trois caractéristiques: ils correspondent à un tour négatif ou interrogatif (la forme affirmative n'étant pas exclue, mais contrainte), et ils sont compatibles avec un gradient : cela a peu / n'a guère de sens; cela renvoie à un type de référent spécifique. Il s'agit d'une décision, d'un jugement, d'un comportement, d'une initiative. (Ex. : cela n'a pas de sens de partir aujourd'bui).

Bon sens apparait dans des constructions qui lui sont propres : d'une décision aussi bien que d'une personne, on peut notamment dire qu'elle est pleine de bon sens / ne manque pas de bon sens.

\subsubsection{A mon sens}

À mon sens (proche de selon moi, selon mon entendement) peut s'analyser comme renvoyant à ma capacité de me donner en propre (i.e. de m'approprier) une représentation d'une situation prise comme déclenchant la mise en œuvre de cette capacité. Un état de choses fait sens à travers le sens que je lui confère.

\subsubsection{Emplois où sens est apparenté à direction}

Il s'agit des emplois de sens dans des séquences du type : (suivre le) sens giratoire; ca part dans tous les sens; mettre dans le bon sens; un palindrome se lit dans les deux sens.

Ces emplois appellent deux remarques :

$1^{\circ}$ Ils mettent en avant la délicate question de l'origine du mot sens. Rappelons qu'ont fusionné en une seule forme deux termes d'origine plus ou moins distincte :

- d'une part, le mot issu du latin sensus, qui désigne, selon les dictionnaires, «l'action de sentir»;

- d'autre part, le mot sinno, issu d'un germanique, qui renvoie à « direction». 
Jean-Jacques Franckel

On peut considérer que la rencontre de ces deux unités, à l'origine distinctes, s'est soldée, du fait de leur affinité profonde, par une forme d'assimilation qui autorise à considérer comme valide une analyse unitaire ${ }^{18}$.

$2^{\circ}$ On observe que sens est ici indissociable d'une circulation; et surtout que cette circulation est toujours inversable et symétrisable, elle s'inscrit toujours dans une relation de complémentarité. Le sens trigonométrique s'oppose au sens des aiguilles d'une montre, le sens giratoire bloque l'autre sens possible, le sens de la marche oppose et symétrise le devant et le derrière ; une voie à double sens est une voie sur laquelle on peut circuler aussi bien dans un sens que dans l'autre: le bon sens (comme façon de disposer un objet) est le symétrique du mauvais sens ${ }^{19}$. De cette donnée, on peut tenir pour emblématique l'expression mettre sens dessus dessous, dans laquelle s'entendent clairement le mouvement et la complémentarité.

\subsubsection{Sens de $Y$}

Les emplois de sens se trouvent massivement associés à la construction prépositionnelle sens de $y$. L'un des enjeux plus généraux du passage d'une forme verbale (sentir) à une forme nominale tient au mode de correspondance entre les paramètres de la forme schématique et les termes constituant le co-texte du mot. Nous avons insisté sur le fait que cette correspondance n'est pas stable: les termes correspondant à ces paramètres peuvent être explicitement présents ou non dans le co-texte et remplir en outre des fonctions syntaxiques variables. Dans le cas du verbe, nous avons vu que cette correspondance peut mettre en jeu des termes renvoyant au sujet syntaxique ou aux différents types de compléments. Dans le cas du nominal, cette mise en jeu d'unités du co-texte passe de façon privilégiée par ce que l'on appelle couramment les « compléments du nom », c'est-à-dire des termes articulés au nom par des prépositions. C'est le cas d'une grande partie des emplois de sens qui s'avèrent indissociables de la préposition $d e$, dans le schéma que nous noterons sens de $Y$, où, comme nous allons le voir, $\mathrm{Y}$ peut avoir des statuts variables. Ce tour permet de façon moins compacte et par conséquent plus analytique que dans les emplois absolus de sens, de préparer le travail de mise en évidence des paramètres de la forme schématique dont nous défendons l'hypothèse qu'ils sont les mêmes que celle du verbe sentir, avant de déterminer par la suite pour chaque emploi les éléments de la séquence correspondant à ces paramètres.

\footnotetext{
${ }^{18}$ D'autant que la distinction ci-dessus n'est pas absolument claire. Selon le RHLF, par exemple, « le français sens a hérité de la polysémie de sensus, à laquelle s'est ajoutée l'influence du mot germanique qui a fourni sens "direction" ». Ce dernier est, selon la même source «issu (v.1130) d'un germanique sinno "direction" et abstraitement, par l'idée de bonne direction, "entendement, raison, intelligence", représenté par le gothique sinba, l'ancien haut allemand sindo "voyager", et qui se rattache peut-être à la famille du latin sensus. Le mot d'abord écrit sen, a été influencé dans son orthographe par sens (issu de sensus) proche sémantiquement pour certains emplois». Sur ce point, cf. aussi G. Merk (1981).

${ }^{19}$ On observe que dans tous ces emplois, la substitution à sens de direction serait impossible.
} 


\subsubsection{Le sens de l'olfaction}

Cet emploi est assimilable dans son principe aux emplois absolus évoqués dans le paragraphe précédent: les cinq sens, l'empire des sens. Olfaction est le nom d'un sens. Comme on l'a vu, sens est ce qui permet à un stimulus $\mathbf{d}$, de rendre $\mathbf{S}$ capable de construire pour lui-même et de s'approprier (sur le mode olfactif) les déterminations du contenu qualitatif $(\mathbf{P})$ de ce quelque chose : ce qu'est / comment est / en quoi consiste ce quelque chose.

\subsubsection{Le sens d'un mot / ce mot a le sens de Y / « tenir» dans le} sens de « résister »

Un existant tangible (un «signifiant») déclenche une représentation mentale intangible et intelligible (un «signifié »). On peut reprendre la glose précédente : sens est ce qui permet à un mot $(\mathbf{d})$ de rendre $\mathbf{S}$ capable de construire pour lui-même et de s'approprier les déterminations du contenu qualitatif $(\mathbf{P})$ de ce quelque chose : ce qu'est / comment est / en quoi consiste ce quelque chose. Un mot a la capacité de rendre le sujet à même de se donner représentation appropriable (ici intelligible) de son contenu ${ }^{20}$. Ce qui diffère dans le cas de «tenir » dans le sens de «résister » est le rôle que joue le terme correspondant à $\mathrm{Y}$ dans le schéma sens de $Y$ : «résister » renvoie ici non plus à $\mathbf{d}$, comme dans le cas de sens d'un mot, mais au contenu qualitatif $\mathbf{P}$ conféré à d.

\subsubsection{Le sens de l'humour, du rythme}

Dans un exemple comme Jean a le sens de l'bumour, la capacité de Jean à mettre en œuvre une représentation et une appropriation de ce qui acquiert la capacité de la déclencher (l'humour) est rendue manifeste. La représentation que manifeste ainsi Jean de l'humour est conforme à l'idée que l'on (en l'occurrence le sujet énonciateur) se fait, de son côté, de l'humour.

\subsubsection{Le sens de la marche / le sens de la hauteur}

Ce type d'emploi a pour caractéristique essentielle 1) de mettre en jeu un mouvement, une circulation: le sens de la marche concerne la circulation d'un véhicule en tant qu'elle met en jeu la capacité d'un sujet à se représenter sa propre orientation en fonction de celle de la marche ; 2) d'inscrire le sens dans une complémentarité : tout comme dans le cas du sens giratoire ou du sens trigonométrique, il est le complémentaire de son inverse.

Dans le cas du sens de la hauteur, nous avons observé que cette séquence impose la préposition dans, et, qu'à son tour, la séquence dans le sens de la hauteur implique un processus finalisé. Le sens de la hauteur correspond à ce que le sujet veut faire, par exemple : passer l'armoire dans le sens de la hauteur.

\footnotetext{
${ }^{20}$ Je définirais la sémantique comme l'analyse des représentations mentales déclenchées par et appréhendées à travers le matériau verbal qui leur donne corps.
} 
Jean-Jacques Franckel

\subsection{Caractérisation de sens}

Cette exploration conduit à une synthèse en termes de forme schématique :

Sens est ce qui permet à quelque chose (d) de rendre $\mathbf{S}$ en mesure de construire et de s'approprier (= d'en faire quelque chose) les déterminations du contenu qualitatif (P) de ce quelque chose : ce qu'est / comment est / en quoi consiste ce quelque chose.

A nouveau, il s'agit par cette forme schématique, non pas d'enfermer le mot dans une définition qui le fige, mais de configurer un espace de déploiement de sa variation.

\subsection{Enjeux de la différence entre sentir et sens}

Nous justifions la légitimité d'une analyse conjointe de sentir et de sens par le fait que leur caractérisation met en jeu, mais dans une configuration différente, les mêmes paramètres ( $\mathbf{d}, \mathbf{P}$ et $\mathbf{S})$.

La différence spécifique entre sentir et sens est sous-tendue par celle plus générale que leur confère leur appartenance catégorielle. On peut résumer un aspect central de cette différence par le fait qu'un nom nomme, et qu'il nomme ce qu'il nomme, ce qui implique la préconstruction de l'existant (au sens large, objectal, idéel, phénoménal, etc.) qui est nommé.

Sentir renvoyait à une double prédication d'existence. Sens renvoie à une double capacité, prise dans une réciprocité: d'une part la capacité de quelque chose (un préconstruit dont sens ne construit pas l'existence) à prendre une fonction de déclencheur (d), et de l'autre la capacité du sujet à construire les déterminations qualitatives du contenu de ce quelque chose (déterminer ce que c'est, comment c'est). Cette capacité n'est pas en propre celle de $\mathbf{d}$ ou celle de $\mathbf{S}$, elle est conférée à la fois à $\mathbf{d}$ et à $\mathbf{S}$. La représentation de $\mathbf{d}$ s'impose au sujet dans le cas de sentir, il devient du pouvoir du sujet de l'avoir et de se l'approprier dans le cas de sens. En résulte une dimension potentiellement téléonomique de sens, qui n'est pas présente dans le cas du verbe sentir.

\subsection{Correspondance entre paramètres de la forme schématique et termes de la séquence}

\subsubsection{Le sens de l'olfaction}

- Y ('olfaction) n'est ni d, ni P. Y est le nom du sens, le nom de ce par quoi (et du mode particulier, inscrit dans un paradigme (les cinq sens), par lequel) un stimulus rend à même un sujet d'avoir la capacité de déterminer de quoi il s'agit, et de se le rendre appropriable.

$-\mathbf{d}$ un stimulus.

- P est le mode (dont Y est le nom) selon lequel ce stimulus devient perceptible, appropriable (sur un mode particulier, nommé olfaction).

$-\mathbf{S}$ est un sujet générique.

\subsubsection{Ce mot a le sens de $Y$}

- Y correspond à $\mathbf{P}$.

- $\mathbf{d}$ correspond au sujet syntaxique (ce mot; « tenir »).

- $\mathbf{S}$ est ici un $\mathbf{S}$ générique, non spécifié, non singulier. 
Sentir / sens

\subsubsection{Le sens de l'bumour, du rythme}

- Y correspond à la fois à $\mathbf{d}$ et $\mathbf{P}$ : le rythme a la capacité de rendre $\mathbf{S}$ à même d'avoir une représentation appropriée de être rythme.

$-\mathbf{S}$ est un sujet spécifique (Jean).

\subsubsection{Le sens de la marche, des aiguilles d'une montre}

- $\mathbf{d}$ est une circulation, un mouvement orienté (celui d'un véhicule, des aiguilles d'une montre).

- ce mouvement orienté rend $\mathbf{S}$ capable d'avoir une représentation de cette orientation en fonction de sa propre orientation et de sa finalisation (le sujet veut ou ne veut pas se mettre dans le sens de la marche, se donne comme orientation de référence celle des aiguilles d'une montre). Le sens est alors dans une relation de complémentarité à un autre sens.

\subsection{Organisation de la variation à partir de la forme schématique}

La double capacité dont relève sens permet de distinguer trois grands cas :

\subsubsection{Actualisation}

Cette double capacité est établie en bloc, à travers son actualisation. C'est le cas où sens renvoie au mode du perceptif, par exemple le sens de l'olfaction. Dans la mesure de l'existence d'un stimulus (d), celui-ci a la capacité de déclencher une forme de représentation que le sujet a dans le même temps la capacité de lui conférer : le sujet ne sent ce qu'il sent que dans l'instant où s'actualise ce double processus. On est alors dans une problématique de l'actualisation: d n'est déclencheur que dans la stricte mesure où s'actualise le déclenchement d'une représentation qui, de son côté, n'a de statut qu'à travers cette actualisation. Cette problématique débouche, du côté du sujet, sur une capacité purement physiologique, sur un effet de l'immédiat (que nous retrouvons certainement dans le mot sensation).

\subsubsection{Adéquation}

d est muni de propriétés qui lui confèrent sa capacité de déclencheur, et qui sont premières par rapport à celles qu'il rend $\mathbf{S}$ en mesure de construire. On est alors dans une problématique de l'adéquation : il est possible de confronter les propriétés de $\mathbf{d}$ en tant que déclencheur et $\mathbf{P}$ : par raccourci, $\mathbf{P}$ est un sens qui peut être confronté au sens de $\mathbf{d}$.

On distingue ici deux cas :

\subsubsection{Le sens des mots et des textes}

Le déclencheur $\mathbf{d}$ correspond à un terme qui est muni d'un contenu qualitatif, et qui a pour fonction intrinsèque d'être le déclencheur de la capacité d'un sujet de construire pour lui-même les déterminations qualitatives de $\mathbf{d}$. C'est par exemple le cas d'un mot, d'un texte, d'une histoire, et plus généralement de tout système sémiologique. Un mot possède un sens en même temps que, et dans la mesure où, 


\section{Jean-Jacques Franckel}

s'agissant de sens, il rend le sujet qui le comprend ou qui l'emploie en mesure de s'en donner une représentation. Cette représentation est alors de l'ordre d'une intelligibilité, étant déclenchée par un élément ayant par lui-même vocation à être intelligible. L'appropriation est de l'ordre du vouloir dire. Le rapport d'adéquation qui s'établit alors se traduit par des formes d'ajustement entre le vouloir dire du sujet et le vouloir dire des mots. L'adéquation peut être plus ou moins bonne : on parlera par exemple du vrai sens du mot, d'un sens dérivé, figuré, erroné, on pourra jouer sur le sens des mots. Un texte ou un signe peut n'avoir pas de sens, ou n'avoir pas beaucoup de sens. Ce qui n'a guère de sens a une faible capacité à rendre $\mathbf{S}$ en mesure d'en avoir une représentation appropriable. Mais quelque chose peut aussi prendre tout son sens sous tel ou tel éclairage.

\subsubsection{Le sens de la marche}

Dès lors qu'il s'agit du sens de la marche, celle-ci est prise comme munie d'un sens intrinsèque ${ }^{21}$, elle définit son propre sens, celui-là même, justement qu'on appelle le sens de la marche (il n'y en a qu'un), et qui s'oppose à son complémentaire. La marche relève ici d'une circulation (la marche d'un véhicule), d'un mouvement linéaire et orienté, par rapport auquel s'ajuste l'orientation du sujet ${ }^{22}$. Celui-ci se donne une représentation de l'orientation qui est celle de la marche, qu'il s'approprie en fonction de sa propre orientation (soit pour s'y conformer, la suivre, soit pour s'y opposer, s'agissant alors d'un ajustement en tout ou rien) : le sens par lequel je m'oriente est le

\footnotetext{
${ }^{21}$ Rappelons la différence cruciale entre le sens de la vie et le sens de la marche : la marche a un sens qui est la sienne; en revanche, la vie n'a de sens que celui qu'on lui confère.

${ }^{22}$ Les emplois qui permettent d'apparenter sens et orientation ont la propriété remarquable d'entraîner de façon plus ou moins nécessaire l'emploi de la préposition dans. Si l'on peut demander quel est le sens de la marche, on demandera dans quel sens on va, on remet un objet dans le bon sens, on passe un meuble dans le sens de la hauteur. On a donc affaire à une structure de la forme $X$ dans le sens de $Y$, donc de la forme $\mathrm{X}$ dans $\mathrm{Z}$ si l'on appelle $\mathrm{Z}$ le sens de $\mathrm{Y}$. On repère donc $\mathrm{X}$ par un sens qui n'est pas le sien propre mais celui de $\mathrm{Y}$.

Cet emploi marque d'une part que $\mathrm{X}$ a un sens et que ce sens se trouve repéré (par le biais de la préposition) par un sens extrinsèque, qui est le sens intrinsèque de $\mathrm{Y}$ : passer un meuble dans le sens de la largeur (dans un contexte de déménagement) marque que le passage est déterminé par les dimensions de la porte, en liaison avec l'assignation d'un objectif. Lorsqu'un objet est dans le bon (ou le mauvais) sens, c'est relativement à ce qu'un sujet veut faire avec cet objet, ce sens étant rapporté à une orientation définie par un objectif assigné. L'emploi de dans pourrait s'expliquer, en toute hypothèse, par le fait que le sens de $Y$ aliène (le sens de) X. Cet emploi entre dans l'abondante lignée des emplois de dans où $X$ dans $Z$ marque des formes variables d'aliénation de $\mathrm{X}$ par $\mathrm{Z}$ : occultation de X par Z, enfermement ou impossibilité pour X de sortir de Z (être dans l'embarras, dans l'impossibilité de faire qch, dans une situation difficile, désespérée) : la situation de $\mathrm{X}$ se trouve repérée par une situation qui n'est pas la sienne propre et qui l'aliène, perte d'autonomie ou de contrôle de X dans le cadre de sa relation à Z (commettre l'irréparable dans un accès de fureur, se laisser emporter dans un accès d' enthousiasme). On peut faire l'hypothèse, sans la justifier ici, que dans associe à $Z$ (dans le schéma $X$ dans $Z$ ) une zone telle que rien ne soit en dehors de cette zone (c'est une zone qui a son « dehors-dedans »), une zone dont l'extérieur est vide: $\mathrm{Z}$ excède toute limite (y compris les éventuelles limites de $\mathrm{X}$ ) en l'absorbant.
} 
sens de la marche ou le sens inverse de la marche. Comme on l'a souligné, sens s'inscrit toujours dans une complémentarité du sens avec son inverse ${ }^{23}$.

\subsubsection{Prédication}

Dans ce dernier cas, c'est la capacité d'un sujet à construire une représentation qui est première, et ce qui acquiert la capacité de déclencher cette représentation est second: le déclencheur fait l'objet, en tant qu'il permet de le déclencher, de l'investissement d'une représentation préalable. Un sujet attribue du sens à quelque chose : il donne un sens à quelque chose. Ce cas s'inscrit donc dans une problématique de la prédication d'une propriété.

Nous distinguerons ici trois sous-cas :

\subsubsection{Une décision (initiative, pensée, parole...) pleine de bon sens}

Le sujet énonciateur attribue à la décision la propriété d'avoir du (bon) sens : elle se trouve investie d'une capacité à rendre le sujet en mesure d'en avoir une représentation conforme à ce qu'il juge bon.

\subsubsection{Jean est plein de bon sens}

La représentation $\mathbf{P}$ est déclenchée par un $\mathbf{d}$ générique (toute situation) auquel se trouve conférée la capacité de mettre en œuvre l'aptitude propre à Jean d'avoir cette représentation, qui est jugée bonne par le sujet énonciateur. Il peut aussi s'agir d'un $\mathbf{S}$ générique lorsqu'il est question du bon sens en général (comme «la chose du monde la mieux partagée $)^{24}$.

\subsubsection{Sens est associé à une téléonomie : quel sens donner à sa vie ?}

Le sujet attribue à la vie la propriété d'avoir un sens, lui confère sa capacité à rendre le sujet en mesure d'en avoir une représentation qu'il s'approprie. La différence entre le sens de la vie et le sens de la marche peut être mise en évidence par le fait que le sens de la vie est celui qu'on lui confere, alors que le sens de la marche lui est intrinsèque.

\subsection{Sens et téléologie. En forme de conclusion}

Il se trouve que le sens constitue, directement ou non, le fondement de très nombreuses réflexions philosophiques et l'objet d'innombrables ouvrages. Il se trouve aussi que le sens ne fait en général pas, ou pas véritablement, l'objet de définitions ni même de tentatives de définition. Il s'agit moins, semble-t-il, de se demander frontalement ce qu'est le sens que de le constituer comme lieu de productions discursives à valeur définitionnelle, comme objet ou comme partie prenante de commentaires dont les zones de recouvrement finissent par en constituer une sorte de

${ }^{23}$ Contre sens peut s'appliquer soit à une voie soit à une interprétation. Il s'agit de deux figures de l'adéquation, en l'occurrence de l'inadéquation: la première se définit par une orientation inverse de celle de la circulation régulière ; la seconde par un ratage dans l'ajustement sémantique.

24 On peut quant à son principe associer à ce cas l'expression à mon sens, qui, comme on l'a vu, consiste à conférer mon sens à un état de choses. 


\section{Jean-Jacques Franckel}

glose ramifiée. Nous sommes dans le cas de figure où ce sont les réflexions que suscite ce qui en constitue le point de départ fuyant et incertain qui permettent a posteriori de tenter d'en établir une sorte de paraphrase tentaculaire.

Dans cette nébuleuse, la question de la téléonomie s'avère constituer un enjeu essentiel.

Lorsque le sens se trouve associé à différents types d'activité métalinguistique, comme la sémantique, la définition d'un mot, l'explicitation d'un texte, l'herméneutique, l'exégèse, etc., on peut considérer qu'il s'agit de rendre intelligible et appropriable un matériau verbal, et que ces activités sont ainsi constitutives d'une forme de téléonomie.

La question est plus complexe lorsque sens se situe en deçà ou au delà du domaine verbal, pour apparaitre dans des questions telles que celle du «sens de la vie », du « sens à donner à ses actions », de « la crise du sens dans la société moderne », etc. La composante téléonomique du sens peut alors s'inscrire dans une conception transcendante $v$ simmanente du sens. Une conception transcendante du sens l'associe à une dimension directionnelle: le sens relève d'une aspiration à un but, d'une orientation déterminée par un pôle définissable en soi et indépendamment de ce qui y mène. S'agissant en particulier du sens de la vie, il peut s'interpréter comme une fin ultime, un objectif transcendant dont il définit la trajectoire et la raison d'être. Une conception immanente du sens l'inscrit dans une dynamique dont le but n'est pas externe, mais constitutif de cette dynamique même. Si direction il y a dans un mouvement, une recherche, une quête, c'est ce mouvement même qui en détermine l'orientation en fonction d'une nécessité interne et non d'un but auto-défini et assigné indépendamment.

La caractérisation proposée ci-dessous du mot sens ne préjuge en rien de l'une ou l'autre de ces conceptions. Mais il s'avère que les constructions dans lesquelles il est mis en jeu semblent y renvoyer plus ou moins directement : il est par exemple très différent de ce point de vue de dire que la vie a un sens, ou qu'elle a $d u$ sens.

Entre le sens de l'olfaction, le sens des mots ou d'un texte, le sens que l'on donne à sa vie, le mot sens traverse, sur le terrain philosophique, trois modes fondamentaux de connaissance $^{25}$. On peut remarquer que ces trois emplois du mot sens relèvent en

\footnotetext{
25 En hasardant un prolongement quelque peu spéculatif, on entrevoit la possibilité d'associer au mot sens le point nodal du discours de G. Deleuze dans sa leçon sur Spinoza : Immortalité et éternité (double CD «à voix haute »; Paris Gallimard 2001), consistant à relier trois dimensions de l'individu et trois genres de connaissances : chaque individu 1) possède une infinité de parties extensives, extérieures les unes aux autres; 2) cet ensemble appartient à chacun sous des rapports caractéristiques ; 3) ces rapports caractéristiques expriment un degré de puissance qui constitue une essence singulière. On peut mettre en parallèle trois genres de connaissance : 1) l'ensemble des idées inadéquates : les affects passions, les signes, les idées confuses, les affects qui découlent de ces affections. L'affect passion découle des affects passifs. Ce premier genre est celui de la connaissance imaginaire par expérience vague, rumeur, oui-dire, répétition automatique et anonymat ; 2) le second genre de connaissance est donné par la connaissance des rapports, de leur composition et de leur décomposition. C'est la connaissance universelle et formelle qui situe les choses en rapport les unes avec les autres, en développant des comparaisons, des mesures, des étalonnages ; 3) le troisième genre dépasse la connaissance des rapports et leurs compositions. Connaissance à la fois rationnelle et intuitive, c'est la connaissance de l'essence qui s'exprime dans les rapports. Si des rapports
} 
même temps, sur le terrain de notre analyse linguistique, de trois modes de mise en œuvre de forme schématique du mot: le sens perceptif relève d'une problématique de l'actualisation (une double capacité qui ne s'exerce que dans son actualisation), le sens des mots et des textes d'une problématique de l'adéquation (toute interprétation est un ajustement plus ou moins réussi), et le sens que l'on donne à sa vie relève d'une problématique de la prédication (on confère, précisément, une propriété à la vie).

Inversement, l'absence de sens renvoie au blocage du processus perceptif, à la surdité du non-entendement, ou à la désorientation de l'errance.

\section{RÉFÉRENCES BIBLIOGRAPHIQUES}

CULIOLI, A., 2002, "À propos de même », in Le lexique entre identité et variation, Langue française, $n^{\circ}$ 133. Larousse, 16-27.

DELEUZE, G., 1969, Logique du sens. Paris : Éditions de minuit.

DE VOGUE, S., 1999, "Construction d'une valeur référentielle : entités, qualités, figures », Travaux de linguistique du CERLICO. Presses universitaires de Rennes, 12, 77-106.

FONTANILLE, J., 1999, "Modes du sensible et syntaxe figurative», in Nouveaux actes sémiotiques, 61 à 63, 1-68.

FRANCKEL, J-J., 1994, «Facteurs lexicaux dans l'organisation de la diathèse », in Subjecthood and subjectivity, the status of the subject in linguistic theory. Londres: Ophrys et institut français du Royaume-Uni, 231-250.

FRANCKEL, J-J. et LEBAUD, D., 1995, «Les échappées du verbe sentir », in Langues et langage : problèmes et raisonnement en linguistique, Mélanges offerts à Antoine Culioli. Paris : PUF, 261-277.

\footnotetext{
caractérisent un individu, c'est par ce qu'ils expriment son essence. Une tentative d'articulation aux propriétés du mot sens pourrait conduire à considérer que le premier mode de connaissance relève de «l'empire des sens »: un mode de connaissance qui est de l'ordre du sentir, une représentation frappée de cécité et de passivité. La double capacité que pose le mot sens selon notre hypothèse débouche, du côté du sujet, sur une capacité purement physiologique, sur un effet de l'immédiat. Le second mode de connaissance relèverait du sens associé au vouloir dire, aux rapports entre le vouloir dire des mots, du monde et des locuteurs, rapports qui ressortissent des processus de régulation et d'ajustement que suppose l'activité langagière. En même temps que le sens relève alors d'une activité qui est de l'ordre du rationnel, il signale que cette activité est indissociable de l'opacité par laquelle ces rapports demeurent de l'ordre d'un vouloir dire. La double capacité mise en œuvre par sens débouche sur la tension de ces rapports où l'adéquation demeure un cas particulier de l'inadéquation. Le troisième mode enfin correspondrait à celui par lequel la double capacité de sens active sa dimension téléonomique. C'est, selon Spinoza, le mode par lequel «nous sentons (sic) et nous expérimentons que nous sommes éternels ». L'éternité comme certitude est à la fois le résultat d'une expérimentation et ce qui constitue l'aboutissement vers lequel s'oriente le mouvement de la vie qui permet cette expérimentation.
} 
Jean-Jacques Franckel

FRANCKEL, J.-J., 2001, «De l'interprétation à la glose», Le Gré des langues, nº 16. L'Harmattan, 48-65.

MERK, G., 1981, «Les résultats de lat. semita et de germ. sinno en langue d'oil », in Travaux de linguistique et de littérature, centre de philologie et le littératures romanes de l'Université de Strasbourg, XIX, 1. Klincksieck, 149-155.

PICOCHE, J., 1986, Structure sémantique du lexique français. Paris : Nathan. 